\title{
Synthesis and evaluation of a protic ionic liquid as a multifunctional lubricant additive
}

\author{
Cheng JIANG ${ }^{1,2}$, Yanan WANG ${ }^{1,2}$, Huaigang SU ${ }^{1,2}$, Weimin $\mathbf{L I}^{1,2, *}$, Wenjing LOU $^{1,2, *}$, Xiaobo WANG ${ }^{1,2}$ \\ ${ }^{1}$ State Key Laboratory of Solid Lubrication, Lanzhou Institute of Chemical Physics, Chinese Academy of Sciences, Lanzhou 730000, China \\ ${ }^{2}$ Qingdao Center of Resource Chemistry \& New Materials, Qingdao 266100, China
}

Received: 19 October 2018 / Revised: 24 January 2019 / Accepted: 22 February 2019

(C) The author(s) 2019.

\begin{abstract}
An oil soluble multifunctional protic ionic liquid (IL) was synthesized and its tribological and antioxidant properties in poly alpha olefin (PAO4) were investigated. The tribological results demonstrated that the IL significantly reduced friction and wear of PAO4. The PAO4 blend with IL resulted in an induced oxidation time of 555 min which is 8.2 and 3.5 times higher than that of pure PAO4 and PAO4 with zinc dialkyl dithiophosphate (ZDDP) for the rotating pressure vessel oxidation test. It is likely that free nonylated diphenylamine acted as a radical scavenger to enhance antioxidant performance, while free bis(2-ethylhexyl) phosphate was more prone to adsorb and react with the metal surface to form a phosphorus-rich tribofilm in order to protect the rubbing surface.
\end{abstract}

Keywords: ionic liquid; friction reducing; anti-wear; antioxidant; multifunctional additive

\section{Introduction}

Ionic liquids (ILs) are molten salts at low temperatures $\left(<100{ }^{\circ} \mathrm{C}\right)$ and are usually composed of bulky asymmetric cations and anions [1]. ILs were first reported as a lubricant in 2001 [2]. Since then, a considerable amount of studies has been published regarding ILs. Initially, imidazolium, pyridinium, ammonium, phosphonium, $\mathrm{Tf}_{2} \mathrm{~N}^{-}, \mathrm{FAP}^{-}, \mathrm{BF}_{4}^{-}, \mathrm{PF}_{6}^{-}, \mathrm{Cl}^{-}$, and $\mathrm{Br}^{-}$were the most extensively studied ions for preparing ILs $[3,4]$. These ILs exhibited negligible volatility, high thermal stability, and outstanding tribological behavior, which are highly desirable properties of lubricants used in extreme environments [5-11]. Unfortunately, their relatively high cost, poor compatibility with conventional base oil, and potential corrosion risks are the major obstacles to their real application in tribology.

Recently, various approaches have been developed to address the above listed issues. Qu's group developed a series of oil miscible ILs and investigated their tribological performance as additives in base oil or fully formulated engine oils [12-14]. Fan et al. reported the preparation of in-situ synthesized ILs and showed that they exhibited good thermal and high temperature tribological properties with an easy and low cost fabrication method [15-17]. Cai et al. developed several multifunctional ILs with anti-wear, antioxidant, and anti-corrosion properties [18-20]. These ILs possessed good compatibility with hydrocarbon base oil and were less corrosive to metal surfaces, making them more suitable for use as lubricants.

On the other hand, due to the increasing concern regarding ash generation and catalyst poisoning of zinc dialkyl dithiophosphate (ZDDP), the development of ashless and low phosphorus anti-wear additives is necessary [21]. ILs are considered to be the most promising candidates to replace ZDDP in lubricating oil. However, antioxidant performance, which is almost equally important as the tribological behavior of ZDDP, has typically been ignored when developing ZDDP replacements. In practice, the deterioration of oxidation stability is the primary concern of ZDDP replacements

* Corresponding authors: Weimin LI, E-mail: liwm@licp.cas.cn; Wenjing LOU, E-mail: wjlou@licp.cas.cn 
when formulating engine oils, hydraulic fluids, and greases [22]. Therefore, there is an urgent requirement to develop ashless, less corrosive, low phosphorus, and multifunctional additives with good tribological and antioxidant performance.

In this study, an oil soluble multifunctional protic IL was synthesized by neutralizing bis(2-ethylhexyl) phosphate (HDEHP) and nonylated diphenylamine (NDPA). The thermal stability, solubility, as well as tribological and antioxidant properties of the IL in $\mathrm{PAO} 4$ were investigated. In addition, the anti-oxidation and tribological mechanisms of the prepared IL are discussed based on the experimental results.

\section{Experimental}

\subsection{Materials}

HDEHP was purchased from Heowns Biochemical Technology Co., Ltd. Nonylated diphenylamine (NDPA) with trade name IRGANOX L 67 was obtained from BASF. ZDDP was chosen for comparison and was kindly provided by Wuxi South Petroleum Additive Co., Ltd. The detailed hydrocarbon groups of ZDDP was not clear. Polyalpha olefin (PAO4), synthetic ester (3970), and oil soluble polyalkylene glycol (OSP 32) were purchased from Exxon Mobil, Croda lubricant, and Dow Chemical, respectively. Mineral oil MVI 150 was obtained from China Petroleum \& Chemical Corporation.

\subsection{Synthesis of the IL}

The IL was synthesized by neutralization reaction. In detail, an equimolar amount of NDPA (42.28 g, $0.1 \mathrm{~mol})$ and HDEHP ( $32.24 \mathrm{~g}, 0.1 \mathrm{~mol})$ were mixed at $25 \pm 5{ }^{\circ} \mathrm{C}$, then the temperature was subsequently increased to $80{ }^{\circ} \mathrm{C}$ with continuous stirring. After $8 \mathrm{~h}$, a viscous, dark bronze product was obtained. The chemical structure of the IL and ZDDP are shown in Fig. 1.

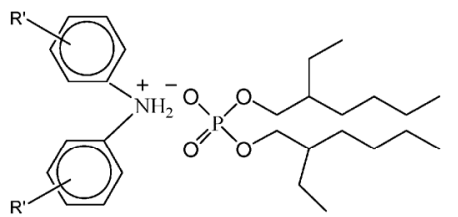

IL

$$
\mathrm{R}^{\prime}=\text { nonyl group } \mathrm{R}=\text { alkyl group }
$$

Fig. 1 Molecular structures of IL and ZDDP.

\subsection{FTIR analysis}

The Fourier transformation infrared (FTIR) spectra of HDEHP, NDPA, and the prepared IL were recorded on a Bruker Tensor 27 FTIR spectrometer from 4,000 to $650 \mathrm{~cm}^{-1}$.

\subsection{Thermal analysis}

Thermogravimetric analysis (TGA) of HDEHP, NDPA, and the prepared IL were measured using a STA 449 TG-DSC instrument. Typically, about $3 \mathrm{mg}$ of the sample was placed in the sample holder and the temperature was raised from 30 to $600{ }^{\circ} \mathrm{C}$ at a heat rate of $10^{\circ} \mathrm{C} / \mathrm{min}$ in nitrogen.

\subsection{Tribological test}

The tribological properties of the prepared IL and ZDDP were performed with a ball-on-disc configuration using an Optimol SRV-V oscillating reciprocating friction and wear tester. A GCr15 bearing steel (AISI-52100) ball with a diameter of $10 \mathrm{~mm}$ and hardness of 58-62HRC was rubbed against a GCr15 bearing steel disc (AISI-52100, 60-64HRC, ø $24.00 \times 7.9 \mathrm{~mm}$ ). The friction and wear tests in this study were conducted at $75^{\circ} \mathrm{C}, 25 \mathrm{~Hz}$ frequency, amplitude of $1 \mathrm{~mm}$, and $100 \mathrm{~N}$ load (corresponding to a calculated initial Hertzian contact pressure of $2.18 \mathrm{GPa}$ ) for a duration of $30 \mathrm{~min}$. The relative humidity was $50 \%-60 \%$. Every test was repeated at least three times to guarantee accuracy. After the tribological tests, a MicroXAM-800 3D noncontact surface mapping profiler was used to measure the wear volume of the worn surface. The morphology and chemical composition of the rubbed surfaces were analyzed using a Hitachi S-3500N scanning electron microscope (SEM) coupled with an energy-dispersive $\mathrm{X}$-ray spectroscopy spectroscope (EDS).

\subsection{Oxidation test}

Pressurized differential scanning calorimetry (PDSC) tests were performed using a NETZSCH DSC 204HP instrument (Bavarian, Germany) according to ASTM D 6186-08(2013). The detailed experimental conditions are described in a previous study [23]. The oxidation onset temperature (OOT) was obtained via temperature programmed experiments and a higher OOT indicates better oxidation stability of the lubricant. The rotating 
pressure vessel oxidation test (RPVOT) was used to evaluate the oxidation stability of the oil in the presence of water and a copper catalyst according to ASTM D2272-14a. Typically, $50 \mathrm{~g}$ of test oil, $5 \mathrm{~g}$ of distilled water, and a fresh copper coil were placed into a glass liner and inserted into the vessel. The vessel was initially pressurized to $600 \mathrm{kPa}$ at room temperature and was axially rotated at $100 \mathrm{rpm}$, at an angle of $30^{\circ}$ from the horizontal in a bath maintained at $150{ }^{\circ} \mathrm{C}$. The results are reported as oxidation induction time (OIT), which was the number of minutes required to reach a $175 \mathrm{kPa}$ pressure loss. A longer OIT suggests improved oxidation stability of the tested oils.

\section{Results}

\subsection{FTIR analysis}

The FTIR spectra of HDEHP, NDPA, and the prepared IL are presented in Fig. 2. The absorption peak at $1,683 \mathrm{~cm}^{-1}$ corresponds to the stretching vibrations of the $-\mathrm{OH}$ group on $\mathrm{O}=\mathrm{P}-\mathrm{OH}$ in HDEHP. The stretching vibration of the $\mathrm{P}=\mathrm{O}$ and $\mathrm{P}-\mathrm{O}-(\mathrm{C})$ bonds in HDEHP and the IL gives rise to corresponding peaks at 1,221 and $1,029 \mathrm{~cm}^{-1}$, respectively [24]. The peaks at 3,406 and $1596 \mathrm{~cm}^{-1}$ can be ascribed to the N-H stretching and bending vibration of NDPA [25]. For the prepared $\mathrm{IL}$, the absence of vibration corresponding to the $-\mathrm{OH}$ group on $\mathrm{O}=\mathrm{P}-\mathrm{OH}\left(1,683 \mathrm{~cm}^{-1}\right)$ indicates that the neutralization process was complete. In addition, the red shift and broadening of the N-H stretching peak at approximately $3,358 \mathrm{~cm}^{-1}$ suggests the formation of

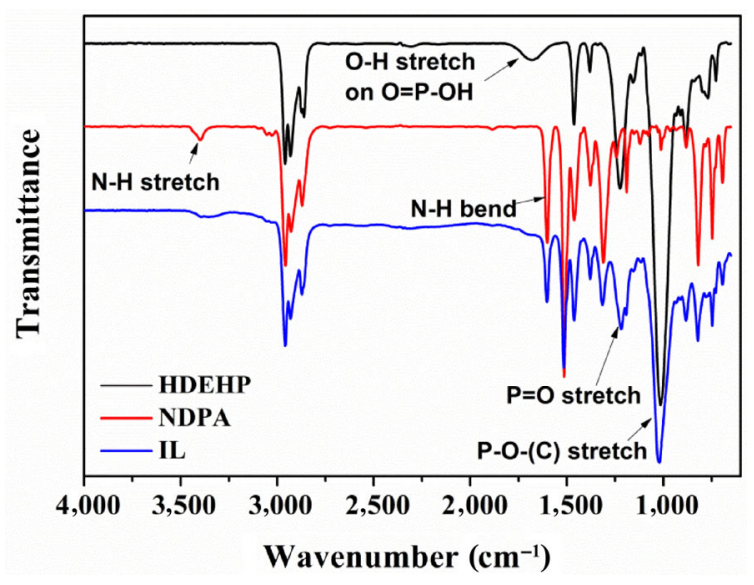

Fig. 2 FTIR spectra of HDEHP, NDPA, and the prepared IL. hydrogen bonds between HDEHP and NDPA, further confirming the formation of the protic IL.

\subsection{Thermal stability}

The TGA curves of the HDEHP, NDPA, and prepared IL are compared in Fig. 3. NDPA showed the highest thermal stability, with an onset decomposition temperature of $368{ }^{\circ} \mathrm{C}$, which is $123^{\circ} \mathrm{C}$ higher than that of HDEHP. For the prepared IL, the onset decomposition temperature was $261{ }^{\circ} \mathrm{C}$, which is $15^{\circ} \mathrm{C}$ higher than that of HDEHP. The intermolecular forces (van der Waals forces and hydrogen bonding) between the anion and cation were likely responsible for the good thermal stability of the IL. It should be noted that the IL TGA curve clearly showed two stages of weight loss. The first stage was the thermal decomposition of the IL anion (DEHP) from 250 to $280{ }^{\circ} \mathrm{C}$ with a rapid weight loss of approximately $43 \%$, in good agreement with the calculated molecular weight of the anion moiety. The second stage exhibited a similar weight loss rate with NDPA and was ascribed to the decomposition of the cation. The onset decomposition temperature of the IL is much higher than that of the traditional anti-wear additive ZDDP, which has been reported to be approximately $200{ }^{\circ} \mathrm{C}$ [26].

\subsection{Solubility of the IL}

Figure 4 shows the images of four base oils (mineral oil, PAO, synthetic ester, PAG) mixed with $5 \mathrm{wt} \%$ of the prepared IL. It is clear that all of blends were clear and no clouds or phase separation was observed, suggesting that the prepared IL exhibited good

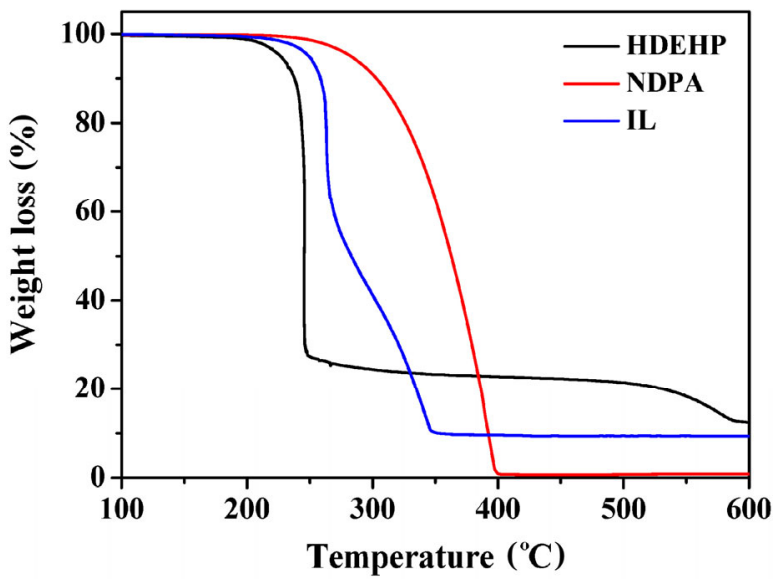

Fig. 3 TG curves of the HDEHP, NDPA, and prepared IL. 


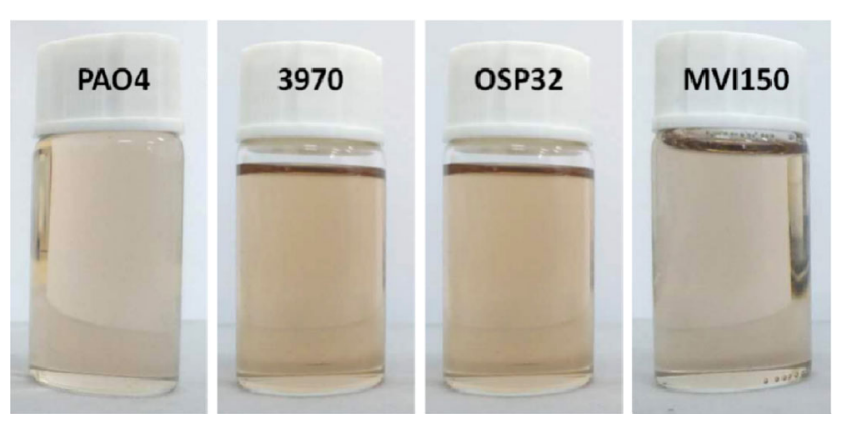

Fig. 4 Solubility of IL in different base oils.

solubility in all base oils. The outstanding solubility of IL in the base oils can be explained by the following reasons. First, the long hydrocarbon chain in the cation and anion moieties enhanced the intermolecular interactions between the IL and base oil, increasing the solubility of IL. Second, the high steric hindrance of the cation and anion led to a weak ion interaction for the IL, which made it less polar than traditional ILs and more compatible with the base oils [27].

\subsection{Tribological performance}

Figure 5 compares the friction coefficients (COF) of the lubricants with different formulations for the ballon-flat reciprocating wear tests. The COF of the PAO4 base oil experienced a high fluctuation for the first $300 \mathrm{~s}$ of sliding which may be due to the high contact stress of the initial test period. The COF subsequently dropped to approximately 0.16 with slight fluctuation. The addition of the IL significantly reduced the COF of the base oil. From Fig. 5, PAO4 with different concentrations of IL showed different running-in performance:

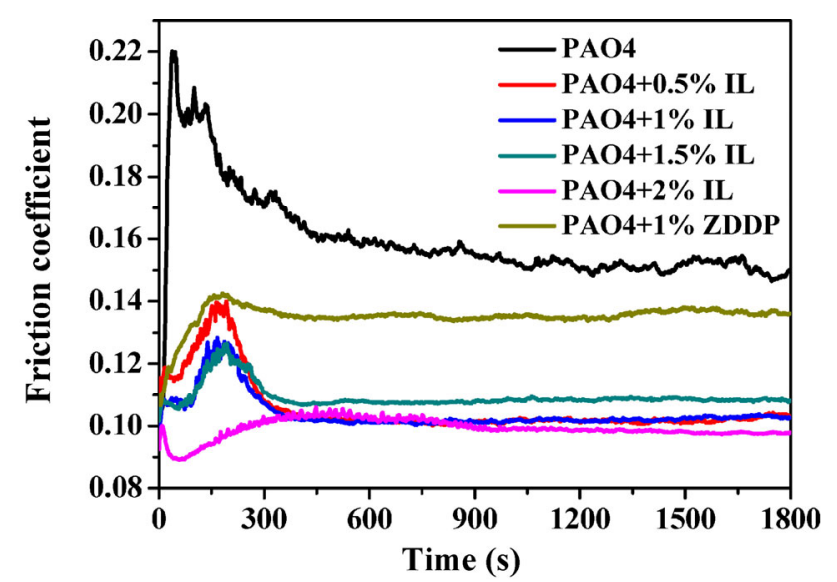

Fig. 5 Variation of the friction coefficient as a function of time with different lubricants .
PAO4 with $0.5 \%, 1 \%$, and $1.5 \%$ IL experienced sharp COF increments during the initial $300 \mathrm{~s}$ running-in period, and subsequently reached low smooth COF values of approximately $0.10-0.11$. When the concentration of IL was increased to $2 \%$, the lubricant showed smooth increases in COF during the running-in period and reached the lowest stable COF of 0.097, indicating superior tribological properties of the IL at this concentration. PAO4 with $2 \%$ IL exhibited the lowest COF among all lubricants tested with a COF reduction of approximately $38 \%$ compared to that of $\mathrm{PAO} 4$, which can be explained by the higher concentration and adsorption between the IL and metal surface. The addition of ZDDP produced a COF of approximately 0.135 which was lower than that of the base oil, but higher than its IL counterpart. The COF of ZDDP was consistent with previous literature values, which showed that the tribofilm formed by ZDDP generally exhibited a high boundary COF ranging from 0.11 to 0.14 [28]. These findings demonstrate that the prepared IL is effective for reducing the friction coefficient of the base oil. The different friction reducing behaviors of IL and ZDDP in PAO are likely related to the nature of the tribofilm formed on the rubbing surface.

Wear volumes of the steel disc after tribological tests are shown in Fig. 6. All wear volumes presented are the averages of tests performed in triplicate. From Fig. 6, PAO4 resulted in the highest wear volume of

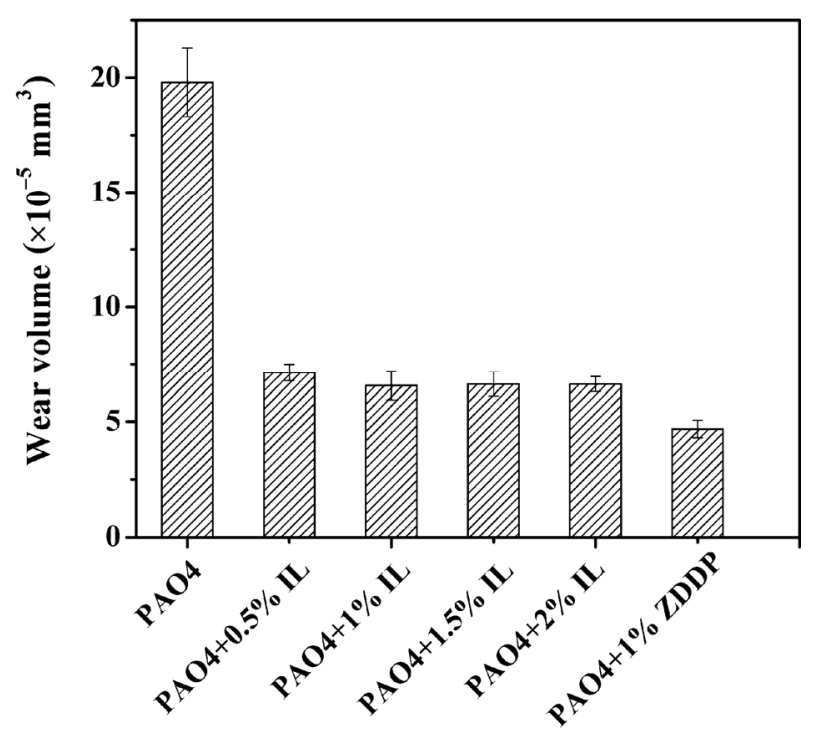

Fig. 6 Wear volume of the steel disc lubricated with the different formulations. 
$19.8 \times 10^{-5} \mathrm{~mm}^{3}$. The addition of anti-wear additives (both IL and ZDDP) greatly reduced the wear volume of the base oil. For the IL, increasing additive concentration from $0.5 \%$ to $2.0 \%$ produced negligible improvement of the wear protection performance, indicating that $0.5 \%$ IL is sufficient to protect the surface from wear. Interestingly, although the friction coefficient of PAO4 with $1 \%$ ZDDP was the highest among all the blended lubricants, the wear volume of ZDDP was $4.68 \times 10^{-5} \mathrm{~mm}^{3}$, which is lower than those of the base oil and IL.

\subsection{Surface characterization}

After the tribological tests, the worn surfaces were ultrasonically cleaned in petroleum ether and examined with SEM and EDS. Figure 7 shows the SEM top view images and EDS spectra of the worn scars lubricated by $\mathrm{PAO} 4, \mathrm{PAO} 4+1 \% \mathrm{IL}$, and PAO4+1\% ZDDP. PAO4 showed the largest wear scar which was accompanied with deep and wide grooves. High magnification images (400×) provided clear evidence of the abrasive and adhesive wear. In contrast, the wear scar generated by PAO4 with IL was much narrower compared to that observed with the base oil. The worn surface was smooth, uniform, and showed no signs of adhesive wear. The worn surface lubricated by PAO4 with ZDDP showed the smallest scar and was interspersed with typical dark patches which were formed by tribochemical reactions between ZDDP and the steel surface [29]. Only Fe and C were detected on the worn surface lubricated by PAO4, which is due to the absence of anti-wear additives. The EDS spectrum of $\mathrm{PAO} 4$ with IL showed clear $\mathrm{O}$ and $\mathrm{P}$ peaks, indicating the formation of a phosphorus-rich tribofilm during the sliding process [27]. The absence of nitrogen on the worn surface lubricated by PAO4 with IL can be explained by either the low activity of nitrogen in the IL or the low sensitivity of light elements in the EDS test. All signature elements ( $\mathrm{Zn}, \mathrm{S}, \mathrm{P}$, and $\mathrm{O}$ ) were detected on the worn surface lubricated by $\mathrm{PAO} 4$ with

(a)
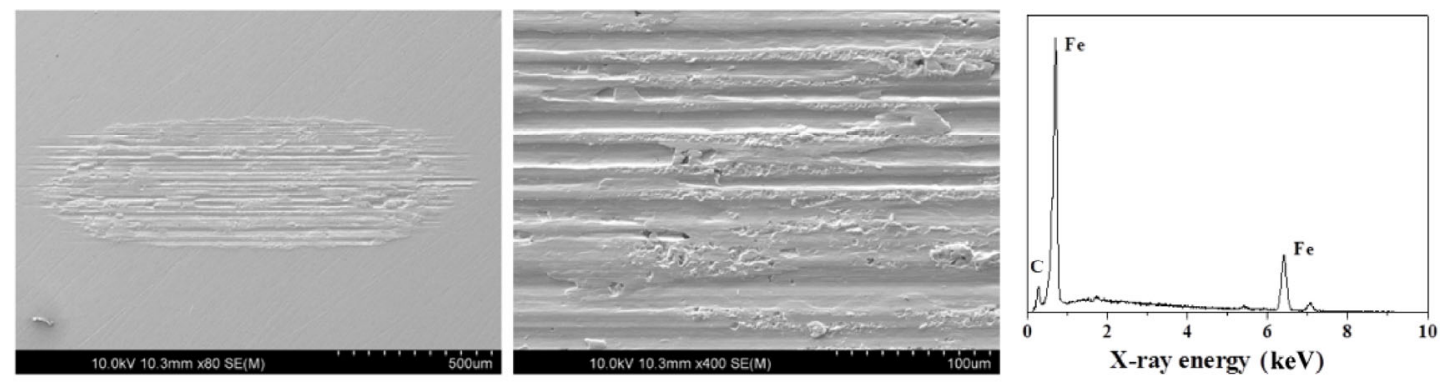

(b)
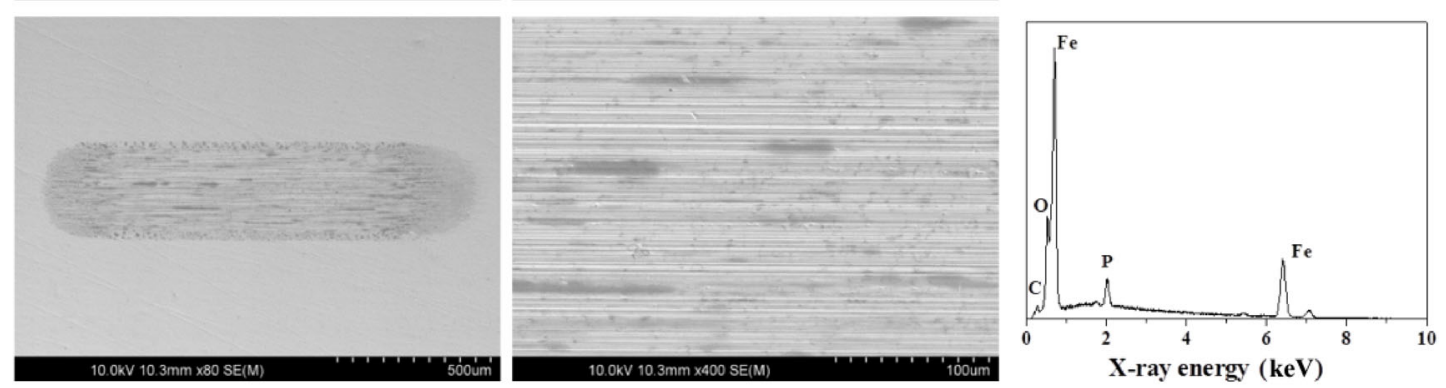

(c)
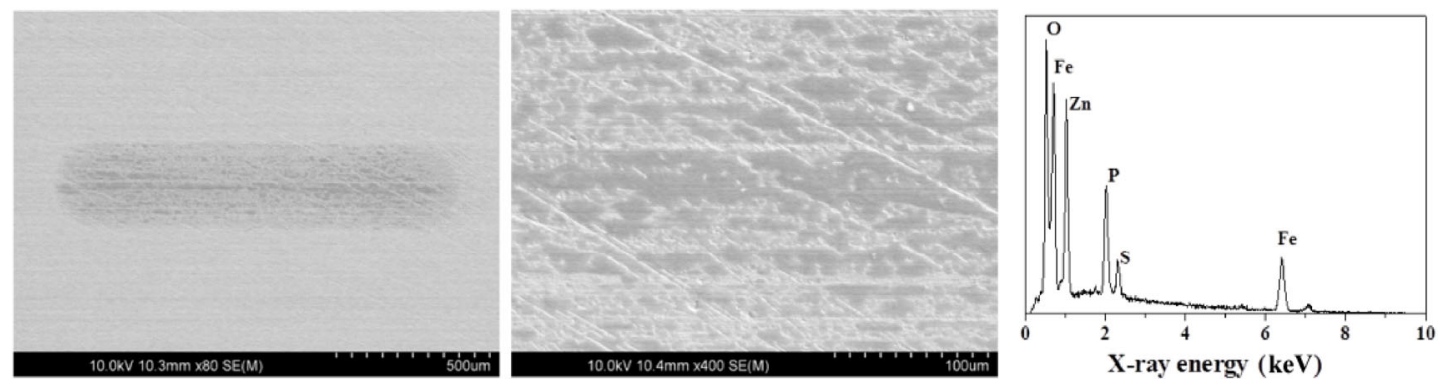

Fig. 7 SEM top view $(80 \times$ and $400 \times)$ and EDS spectra of the wear tracks after the tribological tests. (a) PAO4, (b) PAO4 with $1 \%$ IL, and (c) PAO4 with $1 \%$ ZDDP. 
ZDDP, further indicating the occurrence of tribochemical reactions between ZDDP and the steel surface and formation of the tribofilm. The mechanism of ZDDP on ferrous alloys has been extensively studied. ZDDP can rapidly form a tribofilm with a thickness $>100 \mathrm{~nm}$ with uneven, pad-like distributions separated by deep fissures [29]. This is in good agreement with the results presented in Fig. 7(b) (400×). The tribofilm was mainly composed of closely packed pads of glassy zinc orthophosphate and pyrophosphate and was effective for preventing metal surface contact and reducing adhesive wear [30-33].

\subsection{Antioxidant performance of the prepared IL}

PDSC is a simple and effective method for examining the oxidation stability of lubricating oil. A dynamic model of PDSC was used to measure the oxidation stability of the prepared lubricants, namely the oil samples were placed under oxidizing conditions at a defined heating rate $\left(10{ }^{\circ} \mathrm{C} / \mathrm{min}\right)$ until the onset of the oxidation reaction and the corresponding oxidative onset temperature was recorded. The OOTs of PAO4, PAO4 with 1\% ZDDP, PAO4 with $1 \%$ IL, and PAO4 with $0.57 \%$ NDPA were evaluated by PDSC and the results are shown in Fig. 8. From Fig. 8, the pure base oil exhibited the lowest OOT of $205.3^{\circ} \mathrm{C}$, while the addition of $1 \%$ ZDDP and 1\% IL yielded higher OOT values of 216.6 and $221.4{ }^{\circ} \mathrm{C}$, respectively, suggesting that both ZDDP and IL enhanced the oxidation stability of the base oil. To discuss the anti-oxidation mechanism of IL, the OOT of PAO4 with $0.57 \%$ NDPA (equal mass

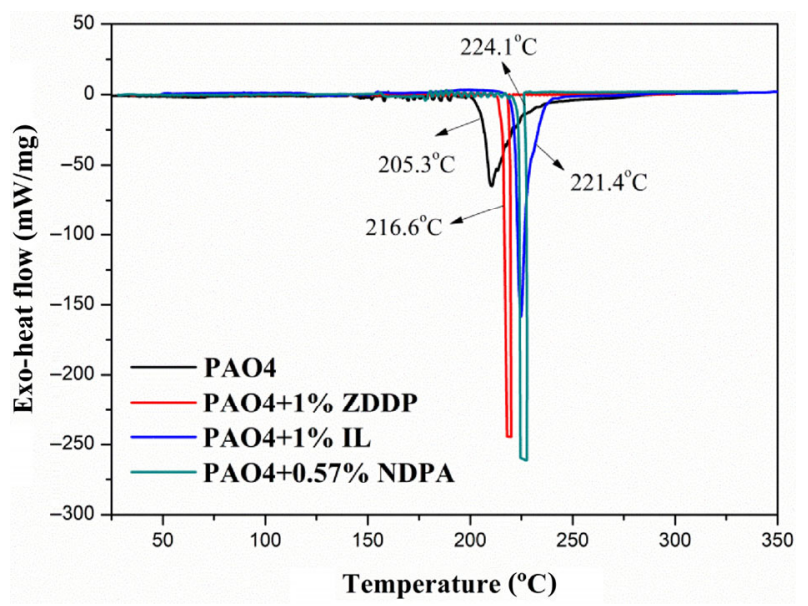

Fig. 8 PDSC curves for the PAO4 andPAO4 with ZDDP, IL, and NDPA. percentage of NDPA in IL) was also investigated. PAO4 with $0.57 \%$ NDPA showed the highest OOT of approximately $224.1^{\circ} \mathrm{C}$, which was higher than those of PAO4 and PAO4 with 1\% ZDDP due to the antioxidation properties of NDPA. For the equal mass percentage of NDPA, PAO4 with $0.57 \%$ NDPA exhibited a slightly higher OOT than that of PAO4 with $1 \%$ IL.

The oxidation stability of $\mathrm{PAO} 4$ and $\mathrm{PAO} 4$ with ZDDP and IL was further examined using the RPVOT and the corresponding oxidation curves are shown in Fig. 9. The RPVOT is widely used to evaluate the oxidation stability of lubricants containing antioxidants in the presence of copper and water as catalysts, which is also effective for distinguishing the antioxidation performance of different antioxidants. The base oil yielded a very low OIT value of $68 \mathrm{~min}$ due to the absence of antioxidant additive. PAO4 with $1 \%$ ZDDP showed an OIT of $159 \mathrm{~min}$, which is 2.3 times longer than that of base oil. Interestingly, the IL additive exhibited exceptional anti-oxidation performance in PAO4 with an OIT of 555 min, which is 8.2 and 3.5 times longer than those of $\mathrm{PAO} 4$ and $\mathrm{PAO} 4$ with $1 \%$ ZDDP, respectively, which is in good agreement with previous PDSC results. The OIT value of PAO4 with $0.57 \%$ NDPA was $765 \mathrm{~min}$, much higher than that of PAO4+1\% IL, which is consistent with the PDSC results. This is likely due to the equilibrium between the IL and free molecules of NDPA and HDEHP. Free NDPA molecules react readily with free radicals to enhance the antioxidant performance.

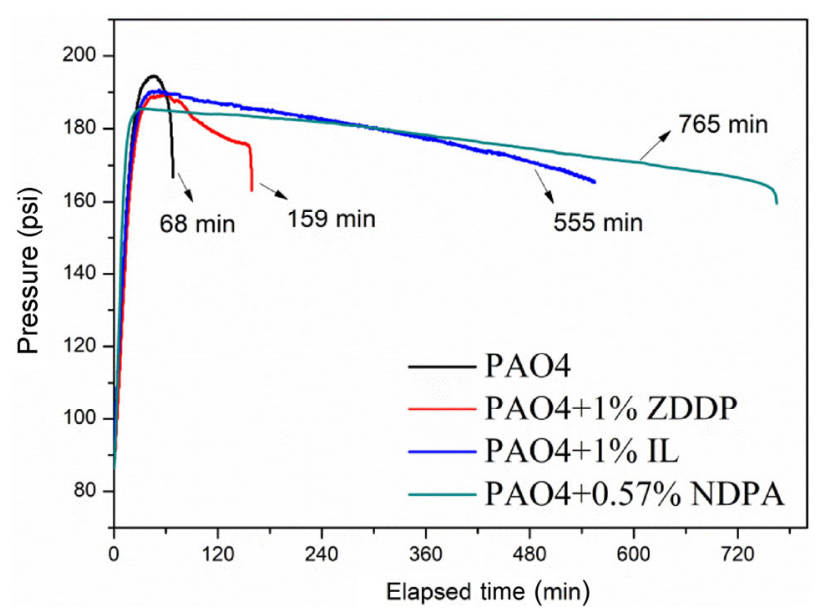

Fig. 9 Oxidation curves of PAO4 and PAO4 with ZDDP, NDPA, and IL. 


\subsection{Antioxidant mechanism of the IL}

It is well-known that the oxidation process of the hydrocarbon base oil involves three basic steps: initiation, propagation, and termination [34]. According to the mechanism, antioxidants can be divided into two categories: primary and secondary antioxidants. Primary antioxidants act as a "radical scavengers" reacting quickly with free radicals during the propagation phase and slowing down the oxidation process by forming new, more stable radicals. Secondary antioxidants react with peroxides and are responsible for breaking the cycle, preventing branching and further propagation [35]. ZDDP is a typical secondary antioxidant additive that reacts with hydroper peroxides and peroxy radicals and is effective for quenching the hydrocarbon oxidation chain reactions [29]. Nevertheless, this process involves chemical breakdown of the ZDDP, resulting in a high consumption rate. In addition, previous studies showed that the oxidation products of ZDDP could exhibit negative effects on tribological performance [29].

The IL fabricated in this study was produced by transferring a proton from HFEHP to NDPA [36]. However, in reality the transform process is likely incomplete. For protic ILs, a dynamic equilibrium exists between the IL and its dissociated forms. In addition, the reaction between the anion and cation is reversible and sensitive to the environment $[37,38]$. As shown in Fig. 10, during the tribological process, free HDEHP tends to absorb onto the metal surface and participates in the tribochemical process to form a protective phosphorus-rich tribofilm. During the oxidation tests, free NDPA more readily reacts with free radical, which possesses higher chemically activity. With the continued consumption of free ions, the equilibrium between the ILs and free molecules is disrupted and an increasing amount of ions will separate from the IL to participate in the tribochemical reaction or act as a free radical scavenger, improving the tribological and antioxidant behavior of the base oil.

\section{Conclusions}

A multifunctional IL with good friction reducing, antiwear, and antioxidant properties was synthesized. The prepared IL exhibited high thermal stability and good solubility in mineral oil, PAO, synthetic esters, and PAG. The tribological results showed that the prepared IL was an effective friction reducing and anti-wear agent in PAO4. The addition of $1 \%$ IL in PAO4 could significantly reduce friction coefficient and wear volume compared to that obtained with the base oil. In addition, both PDSC and RPVOT demonstrate that the prepared IL exhibited better antioxidant properties than that of ZDDP. The dynamic equilibrium between the IL and free reactants was broken with the consumption of HDEHP or NDPA when the oil was subject to frictional or oxidation

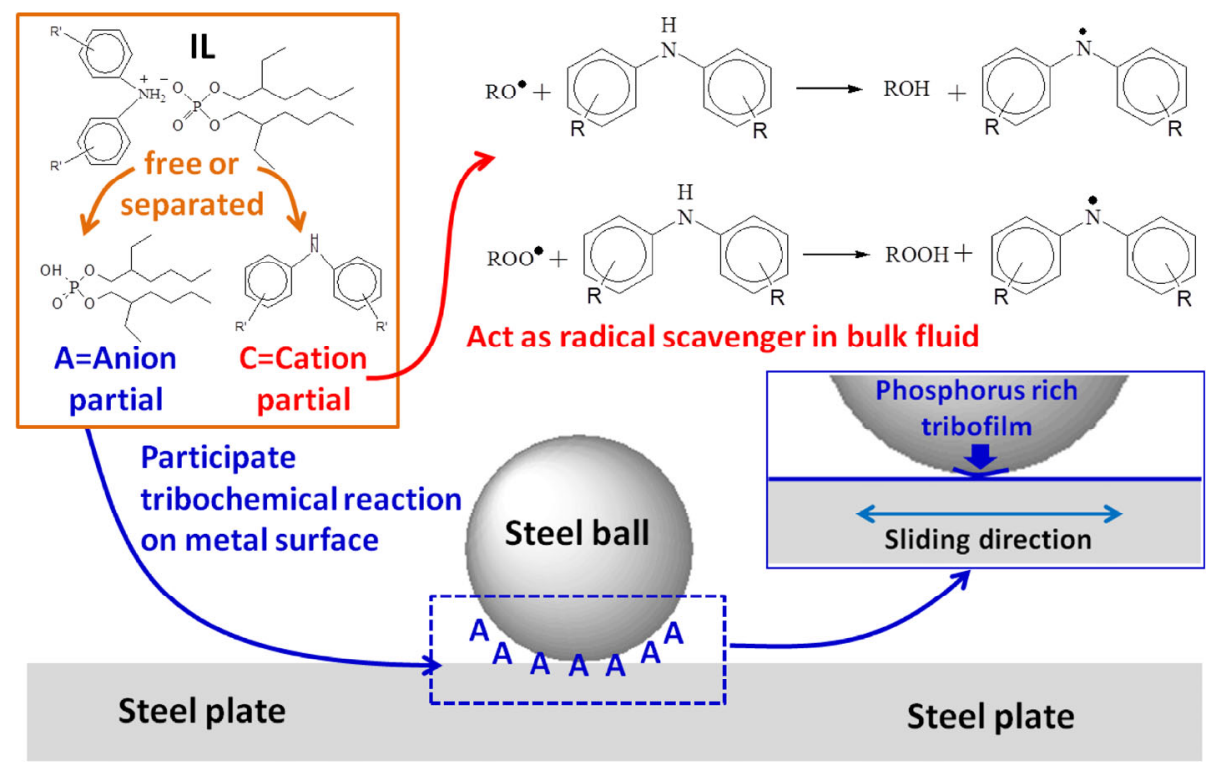

Fig. 10 Schematic diagram of the tribological and antioxidant mechanism of the prepared IL. 
conditions. The free HDEHP participated in the tribochemical reaction with the metal surface, which is responsible for the improvement of the tribological performance. On the other hand, the free NDPA acted as an effective radical scavenger, inhibiting the oxidation of the hydrocarbon chain of the base oil.

\section{Acknowledgements}

The authors gratefully acknowledge the financial support of the National Natural Science Foundation of China (Grant Nos. 51605471, 51505460 and 51775536).

Open Access This article is licensed under a Creative Commons Attribution 4.0 International License, which permits use, sharing, adaptation, distribution and reproduction in any medium or format, as long as you give appropriate credit to the original author(s) and the source, provide a link to the Creative Commons licence, and indicate if changes were made.

The images or other third party material in this article are included in the article's Creative Commons licence, unless indicated otherwise in a credit line to the material. If material is not included in the article's Creative Commons licence and your intended use is not permitted by statutory regulation or exceeds the permitted use, you will need to obtain permission directly from the copyright holder.

To view a copy of this licence, visit http://creativecommons.org/licenses/by/4.0/.

\section{References}

[1] Earle M J, Seddon K R. Ionic liquids. Green solvents for the future. Pure Appl Chem 72(7): 1391-1398 (2000)

[2] Ye C, Liu W, Chen Y, Yu L. Room-temperature ionic liquids: A novel versatile lubricant. Chem Commun 21: 2244-2245 (2001)

[3] Patel D D, Lee J-M. Applications of ionic liquids. Chem $\operatorname{Rec}$ 12(3): 329-355 (2012)

[4] Plechkova N V, Seddon K R. Applications of ionic liquids in the chemical industry. Chem Soc Rev 37(1): 123-150 (2008)

[5] Jiménez A E, Bermúdez M D, Carrión F J, MartínezNicolás G. Room temperature ionic liquids as lubricant additives in steel-aluminium contacts: Influence of sliding velocity, normal load and temperature. Wear 261(3-4): 347-359 (2006)
[6] Jiménez A E, Bermúdez M D, Iglesias P, Carrión F J, Martínez-Nicolás G. 1-n-alkyl-3-methylimidazolium ionic liquids as neat lubricants and lubricant additives in steelaluminium contacts. Wear 260(7-8): 766-782 (2006)

[7] Kamimura H, Kubo T, Minami I, Mori S. Effect and mechanism of additives for ionic liquids as new lubricants. Tribology International 40(4): 620-625 (2007)

[8] Xie G, Wang Q, Si L, Liu S, Li G. Tribological characterization of several silicon-based materials under ionic-liquids lubrication. Tribol Lett 36(3): 247-257 (2009)

[9] Minami I, Inada T, Sasaki R, Nanao H. Tribo-chemistry of phosphonium-derived ionic liquids. Tribol Lett 40(2): 225235 (2010)

[10] Jin C-M, Ye C, Phillips B S, Zabinski J S, Liu X, Liu W, Shreeve JM. Polyethylene glycol functionalized dicationic ionic liquids with alkyl or polyfluoroalkyl substituents as high temperature lubricants. J Mater Chem 16(16): 15291535 (2006)

[11] Jiang C, Li W, Nian J, Lou W, Wang X. Tribological evaluation of environmentally friendly ionic liquids derived from renewable biomaterials. Friction 6(2): 208-218 (2018)

[12] Yu B, Bansal D G, Qu J, Sun X, Luo H, Dai S, Blau P J, Bunting B G, Mordukhovich G, Smolenski D J. Oil-miscible and non-corrosive phosphonium-based ionic liquids as candidate lubricant additives. Wear 289: 58-64 (2012)

[13] Barnhill W C, Qu J, Luo H, Meyer H M, Ma C, Chi M, Papke B L. Phosphonium-organophosphate ionic liquids as lubricant additives: Effects of cation structure on physicochemical and tribological characteristics. ACS Appl Mater Inter 6(24): 22585-22593 (2014)

[14] Elsentriecy H H, Qu J, Luo H, Meyer H M, Ma C, Chi M. Improving corrosion resistance of AZ31B magnesium alloy via a conversion coating produced by a protic ammoniumphosphate ionic liquid. Thin Solid Films 568: 44-51 (2014)

[15] Fan M, Liang Y, Zhou F, Liu W. Dramatically improved friction reduction and wear resistance by in situ formed ionic liquids. RSC Adv 2(17): 6824-6830 (2012)

[16] Fan M, Song Z, Liang Y, Zhou F, Liu W. In situ formed ionic liquids in synthetic esters for significantly improved lubrication. ACS Appl Mater Inter 4(12): 6683-6689 (2012)

[17] Song Z, Liang Y, Fan M, Zhou F, Liu W. Lithium-based ionic liquids as novel lubricant additives for multiply alkylated cyclopentanes (MACs). Friction 1(3): 222-231 (2013)

[18] Cai M, Liang Y, Zhou F, Liu W. Functional ionic gels formed by supramolecular assembly of a novel low molecular weight anticorrosive/antioxidative gelator. J Mater Chem 21(35): 13399-13405 (2011)

[19] Cai M, Liang Y, Zhou F, Liu W. Anticorrosion imidazolium ionic liquids as the additive in poly(ethylene glycol) for steel/Cu-Sn alloy contacts. Faraday Discuss 156(1): 147157 (2012) 
[20] Cai M, Liang Y, Zhou F, Liu W. A novel imidazolium salt with antioxidation and anticorrosion dual functionalities as the additive in poly(ethylene glycol) for steel/steel contacts. Wear 306(1-2): 197-208 (2013)

[21] Zhang Y, Zeng X, Wu H, Li Z, Ren T, Zhao Y. The tribological chemistry of a novel borate ester additive and its interaction with ZDDP using XANES and XPS. Tribol Lett 53(3): 533-542 (2014)

[22] Rizvi S Q A. A comprehensive review of lubricant chemistry, technology, selection, and design. ASTM International, USA, 2009

[23] Chao M, Li W, Chen L, Wang X. Hindered phenol derivative as a multifunctional additive in lithium complex grease. Ind Eng Chem Res 54(26): 6605-6610 (2015)

[24] Qu J, Barnhill W C, Luo H, Meyer H M, Leonard D N, Landauer A K, Kheireddin B, Gao H, Papke B L, Dai S. Synergistic effects between phosphonium-alkylphosphate ionic liquids and zinc dialkyldithiophosphate (ZDDP) as lubricant additives. Adv Mater 27(32): 4767-4774 (2015)

[25] Zhan W, Tu J S, Qian X Z, Li J, Liu J. Synthesis of butyloctyl-diphenylamine as lubricant antioxidant additive by ionic liquids. Int J Adv Manuf Technol 96(5): 1647-1653 (2018)

[26] Qu J, Luo H M, Chi M F, Ma C, Blau P J, Dai S, Viola M B. Comparison of an oil-miscible ionic liquid and ZDDP as a lubricant anti-wear additive. Tribology International 71: 88-97 (2014)

[27] Zhou Y, Qu J. Ionic liquids as lubricant additives: A review. ACS Appl Mater Inter 9(4): 3209-3222 (2017)

[28] Ratoi M, Niste V B, Alghawel H, Suen Y F, Nelson K. The impact of organic friction modifiers on engine oil tribofilms. $R S C A d v$ 4(9): 4278-4285 (2014)

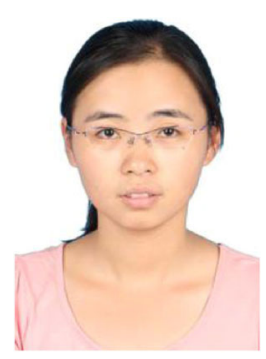

Cheng JIANG. She received her Ph.D. degree in Lanzhou Institute of Chemical Physics, Chinese Academy of Sciences in 2013. She is an assistant research fellow in State Key

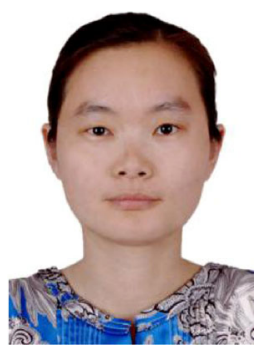

Wenjing LOU. She received her Ph.D. degree in Lanzhou Institute of Chemical Physics, Chinese Academy of Sciences in 2007. She is an associate researcher in the State Key
[29] Spikes H. The history and mechanisms of ZDDP. Tribol Lett 17(3): 469-489 (2004)

[30] Barnes A M, Bartle K D, Thibon V R A. A review of zinc dialkyldithiophosphates (ZDDPs): Characterisation and role in the lubricating oil. Tribology International 34(6): 389-395 (2001)

[31] Topolovec-Miklozic K, Forbus T R, Spikes H A. Film thickness and roughness of ZDDP antiwear films. Tribol Lett 26(2): 161-171 (2007)

[32] Taylor L J, Spikes H A. Friction-enhancing properties of ZDDP antiwear additive: Part I-friction and morphology of ZDDP reaction films. Tribol T 46(3): 303-309 (2003)

[33] Taylor L J, Spikes H A. Friction-enhancing properties of ZDDP antiwear additive: Part II—influence of ZDDP reaction films on EHD lubrication. Tribol T 46(3): 310-314 (2003)

[34] Kawada S, Watanabe S, Tadokoro C, Tsuboi R, Sasaki S. Lubricating mechanism of cyano-based ionic liquids on nascent steel surface. Tribology International 119: 474-480 (2018)

[35] Singh A, Gandra R T, Schneider E W, Biswas S K. Studies on the aging characteristics of base oil with amine based antioxidant in steel-on-steel lubricated sliding. J Phys Chem C 117(4): 1735-1747 (2013)

[36] Greaves T L, Drummond C J. Protic ionic liquids: Properties and applications. Chem Rev 108(1): 206-237 (2007)

[37] Fumino K, Wulf A, Ludwig R. The potential role of hydrogen bonding in aprotic and protic ionic liquids. Phys Chem Chem Phys 11(39): 8790-8794 (2009)

[38] Earle M J, Esperança J M S S, Gilea M A, Canongia Lopes J N, Rebelo L P N, Magee J W, Seddon K R, Widegren J A. The distillation and volatility of ionic liquids. Nature 439: 831 (2006).

Laboratory of Solid Lubrication at Lanzhou Institute of Chemical Physics, Chinese Academy of Sciences. Her research interests mainly focus on high performance lubricants.

Laboratory of Solid Lubrication at Lanzhou Institute of Chemical Physics, Chinese Academy of Sciences. Her research areas include nanofluids, nanoparticle lubricant additives, and high performance lubricants. 